\title{
Economic considerations of breeding for polled dairy cows versus dehorning in the United States
}

\author{
Nathanael M. Thompson, ${ }^{* 1}$ Nicole Olynk Widmar, ${ }^{*}$ Michael M. Schutz, $†$ John B. Cole, $\ddagger$ \\ and Christopher A. Wolf§ \\ *Department of Agricultural Economics, and \\ †Department of Animal Sciences, Purdue University, West Lafayette, IN 47907 \\ fUSDA-Agricultural Research Service, Animal Genomics and Improvement Laboratory, Beltsville, MD 20705-2350 \\ $\S$ Department of Agricultural, Food, and Resource Economics, Michigan State University, East Lansing 48824
}

\section{ABSTRACT}

This paper examines the costs and benefits of selecting for polled dairy heifers versus traditional dehorning practices. Stochastic budgets were developed to analyze the expected costs (EC) associated with polled dairy genetics. The economic assessment was expanded beyond on-farm cash costs by incorporating cost and benefit estimates to generate industry-wide discussion, and preliminary economic evaluations, surrounding the public acceptance and attitude toward polled genetics versus dehorning calves. Triangular distributions, commonly used to represent distributions with limited data, were used to represent labor costs for dehorning, the likelihood of treatment of calf, and the cost of veterinary treatment. In total, 10,000 iterations were run using @Risk v 6.0 (Palisade Corp., Newfield, NY). The EC of the 4 traditional dehorning methods evaluated in this study ranged from $\$ 6$ to $\$ 25 /$ head, with a mean EC around $\$ 12$ to $\$ 13 /$ head. The EC of incorporating polled genetics into a breeding program ranged from $\$ 0$ to $\$ 26 /$ head depending on the additional cost, or premium, associated with polled relative to horned genetics. Estimated breakeven premiums associated with polled genetics indicate that, on average, producers could spend up to $\$ 5.95 /$ head and $\$ 11.90 /$ head more for heterozygous and homozygous polled genetics, respectively, compared with conventional horned genetics (or $\$ 2.08$ and $\$ 4.17 /$ straw of semen at an assumed average conception rate of 35\%). Given the parameters outlined, sensitivity to individual farm semen and dehorning costs are likely to swamp these differences. Beyond on-farm costs, industry-wide discussion may be warranted surrounding the public's acceptance and attitude toward polled genetics versus dehorning or dis-

Received October 4, 2016.

Accepted February 1, 2017.

${ }^{1}$ Corresponding author: thomp530@purdue.edu budding of calves. The value of avoiding dehorning may be larger for the industry, and perhaps some individual farms, than initially suggested if additional value is put on calf comfort and possible worker aversion to dehorning. If public perception of dehorning influences market access, the EC of dehorning may be large but that cost is unknown at present.

Key words: consumer perceptions, cost-benefit, dehorning, polled

\section{INTRODUCTION}

Increasing interest in animal welfare has placed many livestock production practices under enhanced scrutiny. One such practice is dehorning, or disbudding, which is common in both beef and dairy cattle production systems in the United States. According to the American Veterinary Medical Association (AVMA), dehorning cattle conveys a variety of potential benefits, including reduced risk of injury for handlers and other cattle, fewer aggressive behaviors, and reduced feeding trough space (AVMA, 2014). As evidence of these benefits, $94 \%$ of US dairy cattle producers recently indicated routinely dehorning cattle (USDA, 2009).

The majority (68\%) indicated using hot iron disbudding to cauterize horn-producing cells before horn buds attach to the frontal sinus (USDA, 2009). Another $12 \%$ indicated using caustic paste disbudding to chemically destroy the horn-producing cells in the first few days of life (USDA, 2009). Dehorning interventions occurring later in life, specifically scoop or gouge dehorning $(13 \%)$ and saw or wire dehorning $(7 \%)$, were much less prominent given that as horns grow and become attached to the frontal sinus, the procedure becomes more invasive with increased risks of bleeding and infection (USDA, 2009; AVMA, 2014). See Cozzi et al. (2015) for a discussion of the current situation in Europe concerning dehorning.

Despite potential benefits of dehorning noted by the AVMA (2014) and the dairy industry, dehorning also 
results in changes in behavior that have been found to be consistent with acute stress responses from pain (Stock et al., 2013). As a result, these procedures may become a welfare concern for dairy consumers and the public. N. J. O. Widmar, C. Morgan (Purdue University, West Lafayette, IN), C. A. Wolf, E. A. Yeager (Kansas State University, Manhattan), and C. C. Croney (Purdue University; unpublished data) found that dehorning ranked equally with tail docking for the greatest concern, in terms of negative effect on dairy cattle welfare, among 12 dairy production practices investigated. Although dehorning is currently unregulated in the United States, several countries have created dehorning welfare legislation (Stafford and Mellor, 2005). Therefore, despite documented benefits and in light of recent prohibitions on docking tails of dairy cattle in the United States, the risk that consumer acceptance of dehorning will wane is significant.

If dehorning becomes unacceptable in the US marketplace, producers may seek polled dairy cattle. Polled animals have always existed in cattle populations, but intense selection for production attributes in the dairy industry has suppressed polledness in the population, making polled sires rare and often inferior in terms of production relative to their horned counterparts. Nonetheless, the genetic alteration responsible for polledness, contrary to previous speculation, does not appear to affect production performance (Cole et al., 2009; Windig et al., 2015). Incorporating polled genetics into a breeding program has been proposed by the AVMA (2014) as an alternative to dehorning given its potential to eliminate the welfare concerns and expenses associated with dehorning. However, no work has been done to quantify the costs and benefits of this strategy compared with conventional dehorning methods.

The first objective of this work was to develop stochastic cost estimates of selecting for polled dairy heifers versus dehorning. Stochastic budgets were developed to analyze the expected costs (EC) associated with polled dairy genetics. It was hypothesized that sensitivity to individual farm semen and dehorning costs were likely to overwhelm the cost differences between raising or acquiring dairy heifers with polled genetics versus dehorning. The second objective of this work was to expand the economic assessment beyond on-farm cash costs by incorporating both cost and benefit estimates to generate industry-wide discussion, and preliminary economic evaluations, surrounding public acceptance and attitude toward polled genetics versus dehorning calves. In other words, this work sought to determine the economic benefit of using polled genetics if the gap in genetic merit between polled and horned cattle were to disappear.

\section{MATERIALS AND METHODS}

In a simplified version of the decision-making process for dehorning, dairy cattle producers must first decide whether to use traditional dehorning practices to remove horns from calves or to incorporate polled genetics into their breeding program. Decision makers choosing to maintain a traditional dehorning program must then decide which dehorning method to use and whether or not to administer pain relief during the procedure. Producers choosing to use polled genetics face the decision of using a heterozygous or homozygous polled sire. Because polledness is an autosomal dominant trait, $100 \%$ of the offspring from homozygous polled sires will be hornless, regardless of dam genotype. Heterozygous sires, on the other hand, exhibit the polled phenotype but are carriers of the horned gene, creating uncertainty about the phenotype of their offspring (horned or polled).

A series of stochastic partial budgets was developed to simulate costs under 6 potential dehorning/polled genetics scenarios: hot iron dehorning with no pain relief, hot iron dehorning with pain relief, caustic paste dehorning with no pain relief, caustic paste dehorning with pain relief, incorporating homozygous polled genetics into a breeding program, and incorporating heterozygous polled genetics into a breeding program. The costs $\left(C_{j}\right)$ for the first $j=1, \ldots, 4$ traditional dehorning scenarios were estimated as

$$
C_{j}=M C_{j}+P R_{j}+L C_{j}+\left(T R_{j} \times T C\right),
$$

where $M C_{j}$ is materials cost, $P R_{j}$ is the cost of pain relief treatments, $L C_{j}$ is labor cost, $T R_{j}$ is the probability an animal will need to be treated for infection or incomplete dehorning following the procedure, and $T C$ is the cost of this potential follow-up treatment. A different equation was used to estimate the cost of incorporating polled genetics into a breeding program to account for the additional cost, or premium, associated with polled genetics and the uncertainty associated with the phenotype of calves sired by heterozygous sires. The cost $\left(C_{j}\right)$ of the $j=5,6$ polled genetics scenarios was estimated as

$$
C_{j}=P G_{j}+\left(1-p_{j}^{p}\right) \times\left[M C_{l}+P R_{l}+L C_{l}+\left(T R_{l} \times T C\right)\right],
$$

where $P G_{j}$ is the additional cost of polled genetics, $p_{j}^{p}$ is the probability that a calf exhibits the polled phenotype, which is determined by sire and dam genotypes, 
and $M C_{l}, P R_{l}, L C_{l}$, and $T R_{l}$ are the materials cost, pain relief cost, labor cost, and probability of treatment associated with the least-cost traditional dehorning scenario. In other words, when a homozygous polled sire is used $(j=5), 100 \%$ of calves will be born polled (i.e., $p_{5}^{p}=1$ ), and the cost of incorporating polled genetics into the breeding program reduces to $C_{5}=P G_{5}$. However, in the case that a heterozygous polled sire is used $(j=6)$, a portion of calves will be born horned $\left(0<p_{6}^{p}<1\right)$ and incur the cost of traditional dehorning in addition to the cost of heterozygous polled genetics as is indicated in equation [2]. All costs were calculated on a per-calf basis to facilitate comparison across the different dehorning/polled genetics scenarios. Parameter values are reported in Table 1 and are described below.

When estimating costs in equations [1] and [2], $M C_{j}$ and $P R_{j}$ were the only cost parameters known with certainty. Materials cost were $\$ 0.10$ and $\$ 0.15 /$ head for hot iron and caustic paste dehorning, respectively (Payne, 2016). Recommended pain relief treatments for hot iron dehorning included a local anesthetic $(\$ 0.20 /$ head $)$ and a nonsteroidal anti-inflammatory drug (NSAID; $\$ 0.10 /$ head) for a total cost of $\$ 0.30 /$ head (Payne, 2016). On the other hand, the use of a local anesthetic with caustic paste has been found to offer little pain relief and may actually make the procedure more painful (Vickers et al., 2005). Therefore, only the use of a $\$ 0.10 /$ head NSAID is recommended for use with caustic paste.

The remaining cost parameters are unknown when the dehorning/polled genetics strategy decision is made. Therefore, these stochastic variables were best characterized as a distribution of potential outcomes. Stochastic budgeting was used to account for these uncertainties and to give an indication of the distributions of EC for each of the 6 dehorning/polled genetics scenarios. Triangular distributions were used to parameterize $L C_{j}, T R_{j}$, and $T C$. Triangular distributions are often used in such cases with limited sample data because only the minimum, maximum, and most likely values are needed to parameterize the model (Olynk and Wolf, 2009).

At an average employee labor cost of $\$ 14 / \mathrm{h}$ (Ellis and Schulz, 2015), 2 employees working together could perform hot iron or caustic paste dehorning for around

Table 1. Parameter values for deterministic and stochastic dehorning and polled genetics cost variables

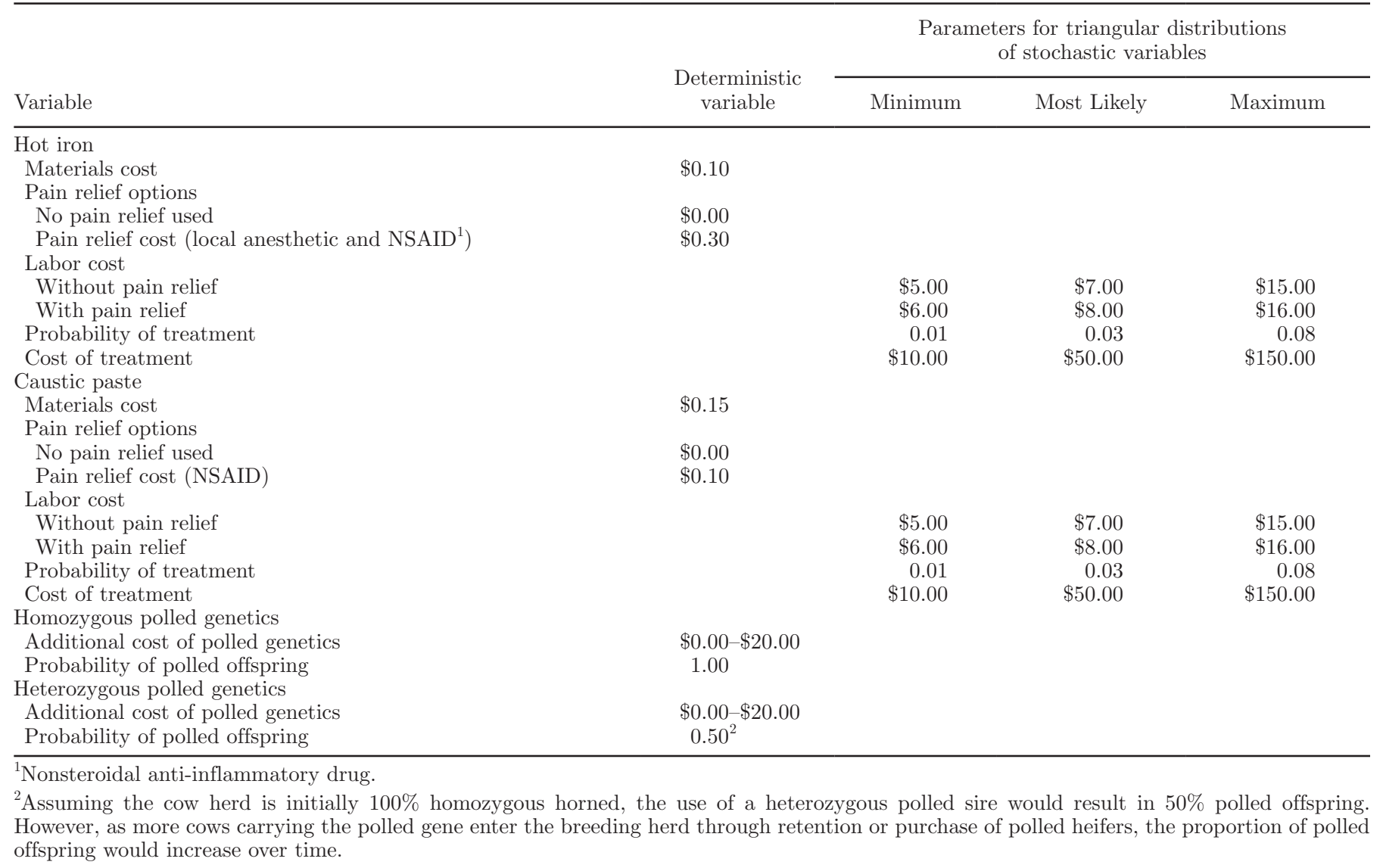


$\$ 7 /$ head. However, the actual cost of labor associated with dehorning is expected to vary with multiple factors, including the cost of labor $(\$ / \mathrm{h})$, experience of the labor, the facilities used to house and restrain calves, the amount of time necessary to gather and then return supplies, and so on. Therefore, the distribution of labor cost for hot iron and caustic paste dehorning was parameterized using a triangular distribution with a minimum value of $\$ 5$, maximum of $\$ 15$, and most likely value of $\$ 7 /$ head.

In addition to the pain relief treatment costs described above, the distribution of labor costs may also be affected by the administration of pain relief. On the one hand, labor costs will be necessarily higher because of the additional time required to administer pain relief. However, if animals become more docile while being dehorned because of pain relief treatments, labor costs may simultaneously decrease. Potential time savings are not documented and are difficult to estimate because they will likely vary by animal and with the particular pain relief treatment administered. Time savings associated with more docile calves would be most likely with administration of a sedative, which was not considered here. For this reason, only the additional time required to administer pain relief treatments was included in our analysis. It is important to note that the amount of time necessary to administer pain relief will vary depending on multiple factors, most notably the facilities used for handling calves. Here, an average of $2.1 \mathrm{~min}$ per shot reported by Olynk and Wolf (2008) was incorporated into the calculation of labor costs. The resulting distribution of labor costs for scenarios with pain relief was parameterized as follows: minimum of $\$ 6$, maximum of $\$ 16$, and most likely value of $\$ 8 /$ head.

Dehorning is a relatively safe procedure if performed by properly trained employees at the earliest possible age - the first 6 to 8 wk of life (AABP, 2014). Nonetheless, the procedure exposes calves to the risk of infection or the possibility of incomplete dehorning, which may require additional treatment. The rate at which animals need to be treated following dehorning is random and can be characterized by a triangular distribution with a minimum value of $1 \%$, a maximum of $8 \%$, and a most likely value of $3 \%$ (Widmar et al., 2013).

Similarly, the cost of these treatments varies depending on the severity of the problem. Therefore, treatment cost was assumed to be triangularly distributed with a minimum value of $\$ 10$, a maximum of $\$ 150$, and a most likely value of $\$ 50 /$ head (Widmar et al., 2013). Treatment costs were admittedly difficult to determine due to the wide range of potential costs across the various potential reasons for requiring treatment, overall health of the calf, varying veterinary expenses across geogra- phies (and even across farms within geographies), calf and heifer health management skill level on the farm, and various other factors. However, we believe that the values used here characterize the most likely, or most reasonable, window of potential treatment costs. For example, one could imagine the minimum of $\$ 10 /$ head representing the most minor of interventions, such as a having a veterinarian consult or having the calf treated by the herdsperson, and the most likely and maximum values of $\$ 50$ and $\$ 150 /$ head represent cases of more intensive treatment or intervention.

Under a scenario in which homozygous polled dairy cattle genetics are incorporated into a breeding program, all calves would be born without horns, and the material and labor costs associated with dehorning would be eliminated completely. Depending on the genotype of the dam, calves sired by heterozygous polled bulls have some chance of being born with horns. Assuming the initial cow herd is $100 \%$ homozygous horned, there is a $50 / 50$ chance that calves will be born polled (Spurlock et al., 2014). Although material and labor costs are eliminated for the $50 \%$ of calves born without horns, the other $50 \%$ will still incur the cost of traditional dehorning intervention.

Further, calves that are born heterozygous polled; that is, they exhibit the polled phenotype but are carriers of the horned gene, have the possibility of having scurs, which are incompletely developed horns attached to the skin rather than the skull that can vary in size from small growths to small horn-like structures (Bullock, 2015). As a result, the potential exists for producers to mistake scurs for small horn buds in young calves, resulting in unnecessary dehorning of some polled animals. The presence or absence of scurs is thought to be controlled by a different gene from the one controlling polledness, but these 2 genes may interact with each other as well as with calf sex. However, the scurred trait is still not completely understood (Bullock, 2015); therefore, modeling it is impractical. As a result, the potential cost associated with unnecessarily dehorning polled animals that have scurs was not included in our analysis.

As interest in polled dairy cattle genetics has grown in recent years, differences in the genetic merit or genetic potential of polled versus horned sires has received a lot of attention. Although polledness does not seem to alter production performance (Cole et al., 2009; Windig et al., 2015), a recent review of AI sires in the United States indicated that not only is there limited availability of bulls carrying the polled gene but, on average, these animals currently sacrifice genetic merit relative to their horned counterparts due to intense selection pressure for production attributes in the dairy industry, in particular in the Holstein breed (Spurlock et 
al., 2014). Thus, the limited number of polled individuals limits the opportunity to identify polled sires with elite genetic merit for production traits. For example, in 2013 the average Net Merit (NM\$) of the top $20 \%$ of Holstein sires was $\$ 778(\mathrm{n}=228), \$ 606(\mathrm{n}=11)$, and $\$ 426(\mathrm{n}=3)$ for horned, heterozygous polled, and homozygous polled sires, respectively (Spurlock et al., 2014). Thus, the economic consequences of incorporating polled genetics into a breeding program will depend on whether heterozygous or homozygous polled sires are used and the rate at which these genetics are incorporated. Evaluating several different breeding strategies, Spurlock et al. (2014) found that incorporating polled genetics over a $10-\mathrm{yr}$ period decreased $\mathrm{NM} \$$ between 5 and $25 \%$. However, overall merit in their study did not account for the value of genetic improvement that would result from increased frequency of the polled gene. Taking into account these potential genetic improvements, Scheper et al. (2016) found that a selection scheme based solely on sire genotype was essential to a fast transition to a completely polled population $(<25$ generations in their study) but was contradictory to the preservation of high genetic potential for milk yield. However, they found that selection based on male genotypes with moderate weighting and female phenotypes with high weighting could be appropriate for meeting both acceptable production levels and increasing the frequency of the polled trait (Scheper et al., 2016).

Dorshorst (2014) contends that because of current, rapid growth in the polled segment of the dairy population, the gap in genetic merit between horned and polled animals will continue to decrease. For example, between 2010 and 2013, the number of polled Holstein bulls registered with the National Association of Animal Breeders (NAAB) increased from 14 to 74 (Spurlock et al., 2014). An evaluation of Dutch and Canadian breed associations yielded similar findings, with the number of polled sires increasing from 33 to more than 150 between 2009 and 2014 (Windig et al., 2015), and a reduction in the overall merit index gap between horned and polled sires. That is, between 2009 and 2014, the gap in total merit index decreased from 180 points, or about 18 years of selection, to 149 points, or just 5 years of genomic selection. [Note: Windig et al.'s (2015) measure of overall merit index is the Dutch-Flemish total merit index (NVI), which combines breeding values for production, longevity, fertility, health, and conformation.]

For the purposes of this analysis, no difference in milk production of the heifers or cows produced with conventional versus polled semen were modeled due to the imperfect relationship between merit, productivity, and profitability in dairy systems. Although genetics clearly contribute to the economic potential of a cow over her lifetime, management factors and other variables also play a role. Given this imperfect relationship, various other factors, and clear trend to lessen the gap in merit between conventional and polled sires, differences in economic performance in the model would be extremely difficult to estimate accurately.

It is also important to note that polled dairy cattle genetics are not costless. That is, holding all else constant, there is an additional cost, or premium, associated with polled genetics relative to horned. Given that the cost of semen is influenced by a variety of different factors; namely, production characteristics, in addition to polledness, this cost can be difficult to quantify and will change over time as the supply and demand for polled genetics evolves, likely converging toward the cost of traditional dehorning methods. As a result, an evaluation of current semen prices offered little insight (STgenetics, 2016). Therefore, rather than identifying a single unreliable value to represent the premium associated with polled genetics, the cost of incorporating polled genetics into a breeding program in our analysis was simulated for additional costs of polled genetics ranging from $\$ 0$ to $\$ 20 /$ head to determine the effect of the polled semen premium on the optimal dehorning strategy.

In addition, the effect of dehorning on production attributes, such as average daily gain or feed intake, is not quantified in equations [1] or [2]. Previous research has indicated that, depending on pain relief treatments, dehorning may reduce weight gain in the days immediately following the procedure (Newton and O'Connor, 2013; AVMA, 2014; Bates et al., 2015). However, Laden et al. (1985) found that the mean BW of Holstein heifer calves that were dehorned were not significantly different than animals that were not dehorned 4 wk after the procedure. Therefore, there does not appear to be any long-term economic consequences from the effect of dehorning on production attributes of calves that should be considered.

With equations [1] and [2] parameterized, @Risk 6.0 (Palisade Corp., Newfield, NY) was used to evaluate stochastic budgets for each of the 6 dehorning/polled genetics strategies. Expected costs were estimated using Monte Carlo integration where @Risk's triangular distribution function was used to generate 10,000 iterations for each of the stochastic parameters. In addition to estimating EC, distributions of costs were evaluated for each dehorning strategy, and breakeven analysis was used to determine the premium a producer could pay for homozygous or heterozygous polled genetics that would make them indifferent to traditional dehorning practices. 


\section{RESULTS AND DISCUSSION}

\section{Cost of Developing Heifers via Alternative Strategies}

Simulated costs for the 4 traditional dehorning methods ranged from $\$ 6$ to $\$ 25 /$ head, with a mean EC around $\$ 12$ to $\$ 13 /$ head (Figure 1). Differences in mean EC were small $(<\$ 1.30 /$ head $)$ given that materials, pain relief, and labor costs were the only parameters that differed between these 4 scenarios. Nonetheless, hot iron disbudding without the use of pain relief was the least-cost traditional dehorning strategy, $\mathrm{EC}=\$ 11.90$ / head. This is consistent with current industry practices, where $68 \%$ of US dairy producers who dehorn their cattle use hot iron disbudding, and of those, only $14 \%$ use any sort of pain relief (analgesics or anesthetics; USDA, 2009).

The cost of pain relief was treated as deterministic in our model and was assumed not to affect the probability of treatment or treatment cost. That is, other than the slightly higher distribution of labor costs associated with administering pain relief and improved animal welfare from reduced pain during and after the procedure, there are no other quantifiable productionrelated benefits or costs to the producer from using pain relief. Therefore, scenarios that include pain relief consistently generate higher costs than scenarios without pain relief. Nonetheless, is it is important to note that should the use of pain relief be mandated by dehorning welfare legislation, as it has been in several other countries (Stafford and Mellor, 2005), hot iron disbudding may no longer be the least-cost traditional dehorning strategy. Instead, given the higher cost of recommended pain relief treatments associated with hot iron disbudding, caustic paste disbudding with pain relief was the least-cost method in our analysis with EC $=\$ 13.04 /$ head.

The costs of incorporating polled genetics into a breeding program were simulated for values of the additional cost of polled genetics ranging from $\$ 0$ to $\$ 20$ / head (Figure 2). Resulting mean EC ranged from $\$ 0$ to $\$ 20 /$ head for homozygous polled genetics and $\$ 6$ to $\$ 26 /$ head for heterozygous polled genetics. Comparing these values with the mean EC of the least-cost traditional dehorning strategy (hot iron, no pain relief, $\mathrm{EC}=\$ 11.90 /$ head$)$, on average, breakeven additional semen costs were $\$ 5.95 /$ head for heterozygous polled genetics and $\$ 11.90 /$ head for homozygous polled genetics. That is, a producer who pays an $\$ 11.90 /$ head (\$5.95/head) premium for homozygous (heterozygous) polled genetics would have an EC equivalent to that of hot iron disbudding with no pain relief, and as a result would be indifferent between the 2 alternatives. Breakeven premiums for polled genetics relative to the other traditional dehorning methods evaluated would be slightly higher $(<\$ 2 /$ head $)$ due to the higher EC associated with these alternatives. However, premiums for polled genetics above breakeven levels would disincentivize the adoption of polled genetics, given that traditional dehorning methods would result in lower EC.

Note however that these breakeven premiums are reported in $\$ /$ head and not $\$ /$ straw of semen. Interpreting the values reported above as $\$ /$ straw of semen would imply an unrealistic conception rate of $100 \%$. There-

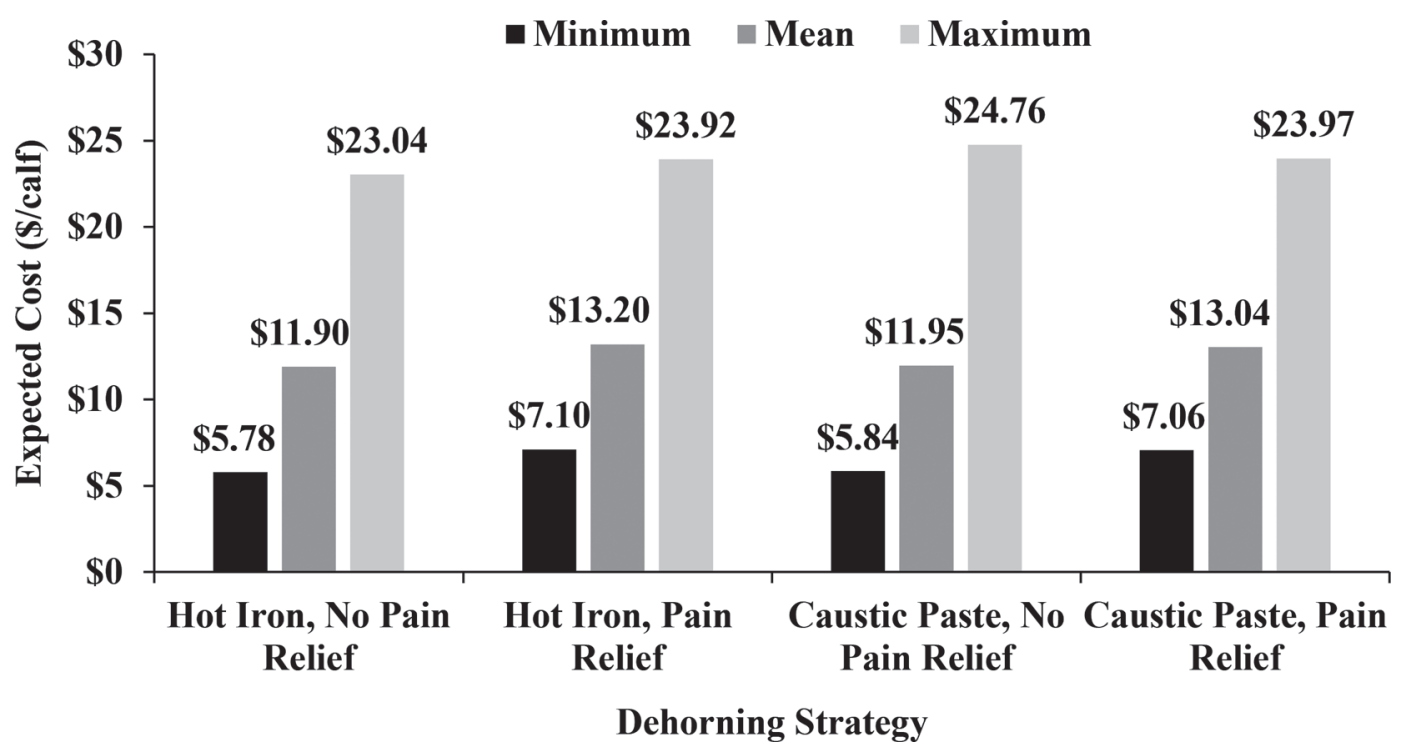

Figure 1. Minimum, mean, and maximum expected costs $(\$ /$ calf $)$ for hot iron and caustic paste dehorning with and without pain relief treatments. 


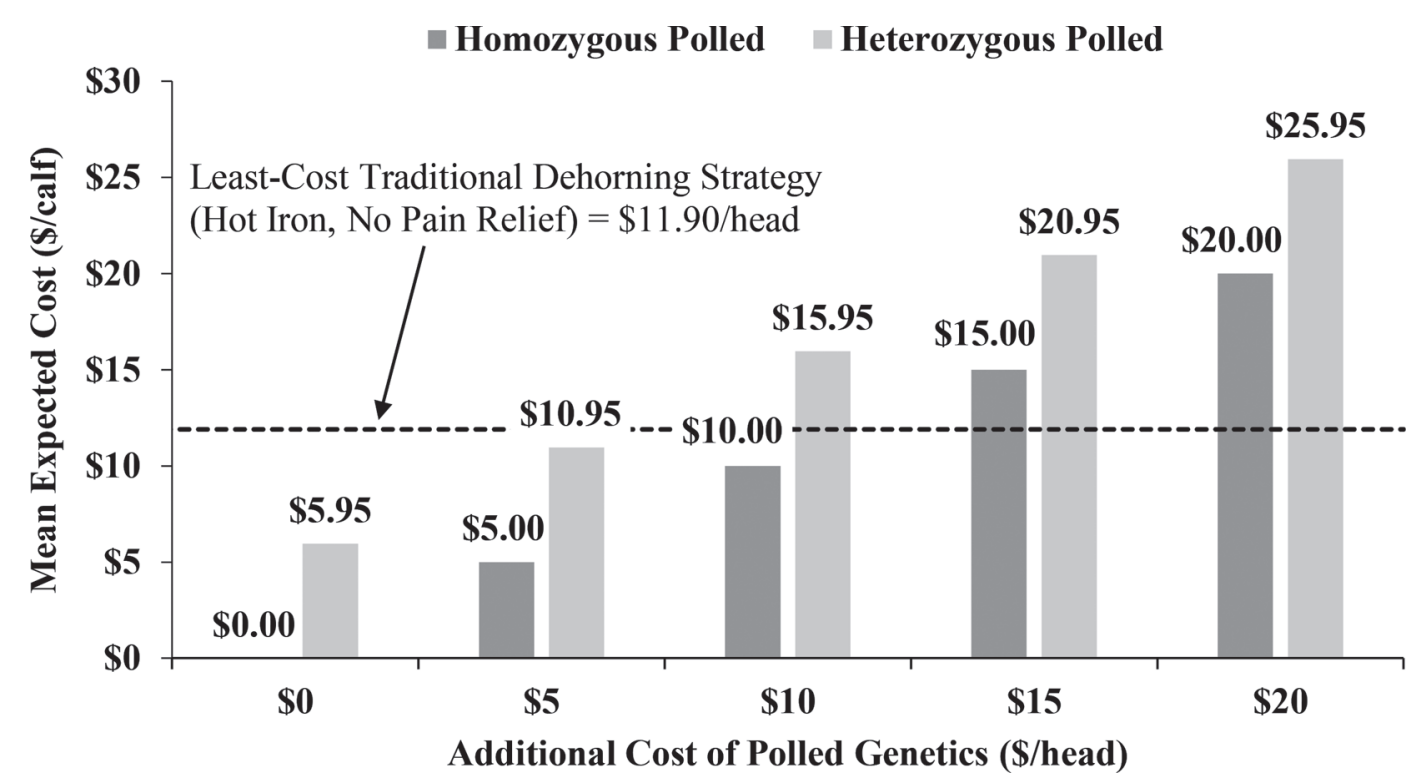

Figure 2. Mean expected costs (\$/calf) of incorporating homozygous and heterozygous polled genetics into a breeding program for 5 values of the additional cost ( $\$ 0$ to $\$ 20 /$ head) of polled genetics.

fore, $\$ /$ head premiums can be converted to $\$ /$ straw of semen by multiplying by the expected conception rate. For example, at an average assumed conception rate of $35 \%$, the average breakeven polled semen premium was $\$ 2.08$ /straw of semen for heterozygous polled genetics and $\$ 4.17 /$ straw of semen for homozygous polled genetics. That is, a producer with an expected conception rate of $35 \%$ would be indifferent to hot iron disbudding with no pain relief at a $\$ 4.17 /$ straw of semen $(\$ 2.08$ / straw of semen) premium for homozygous (heterozygous) polled genetics.

Consider an example where heterozygous and homozygous polled genetics are available at premiums of $\$ 5 /$ head ( $\$ 1.75 /$ straw of semen) and $\$ 12 /$ head $(\$ 4.20$ / straw of semen), respectively, over non-polled semen. Using a decision tree, the EC for each dehorning alternative is estimated, and the decision to incorporate heterozygous polled genetics into the breeding program is identified as the least-cost dehorning strategy, EC $=\$ 10.95 /$ head (Figure 3). This is consistent with the previous discussion of breakeven premiums given that the additional cost of heterozygous polled genetics in this case is less than the estimated breakeven value, resulting in a lower EC than the least-cost traditional dehorning method, (hot iron, no pain relief, EC = $\$ 11.90 /$ head). On the other hand, the additional cost of homozygous polled genetics in this example is higher than the previously estimated breakeven value, resulting in an $\mathrm{EC}=\$ 12.00 /$ head, which is higher than not just the least-cost traditional dehorning method but also the heterozygous polled genetics scenario. That is, even with the added cost of having to dehorn a portion of the offspring sired by heterozygous polled bulls, the much lower additional cost of heterozygous relative to homozygous polled genetics in this case made it the least-cost strategy.

It is important to note that values reported in Figures 2 and 3 are means from distributions of EC. That is, these are the EC that we would expect on average but, in some cases, EC will be higher or lower than these values. For example, the additional cost of heterozygous polled genetics of $\$ 5 /$ head in the previous example would result in lower EC than the least-cost traditional dehorning method only $86 \%$ of the time. Estimated breakeven premiums ranged from $\$ 6$ to $\$ 23$ / head for homozygous polled genetics and $\$ 3$ to $\$ 12$ / head for heterozygous polled genetics (or $\$ 2$ to $\$ 8$ and $\$ 1$ to $\$ 4 /$ straw of semen at an assumed average conception rate of $35 \%$; Figure 4). That is, depending on cost/efficiency of labor, the probability of follow-up treatments, and the cost of treatment, some producers may have higher or lower break-evens in terms of what additional cost they are able to pay for polled genetics relative to using traditional dehorning methods. For example, a producer with low dehorning costs (e.g., low cost or highly efficient labor) would only be able to pay a small premium for either heterozygous $(<\$ 3 /$ head; $<\$ 1 /$ straw of semen) or homozygous $(<\$ 6 /$ head; $<\$ 2 /$ straw of semen) polled genetics before it would become more cost effective to revert to traditional dehorning methods. On the other hand, producers with high dehorning costs may be able to pay as much as an 
THOMPSON ET AL.

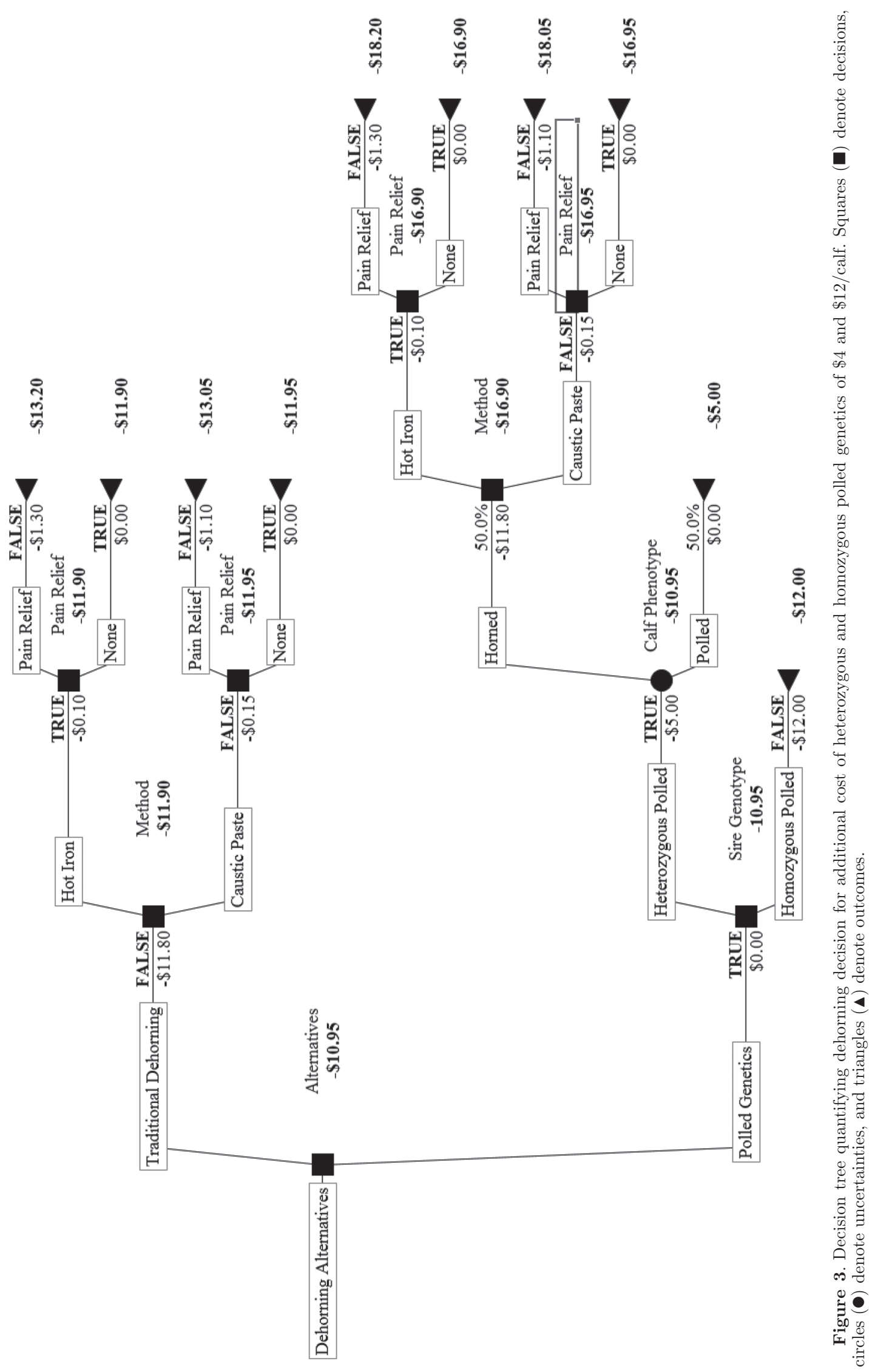




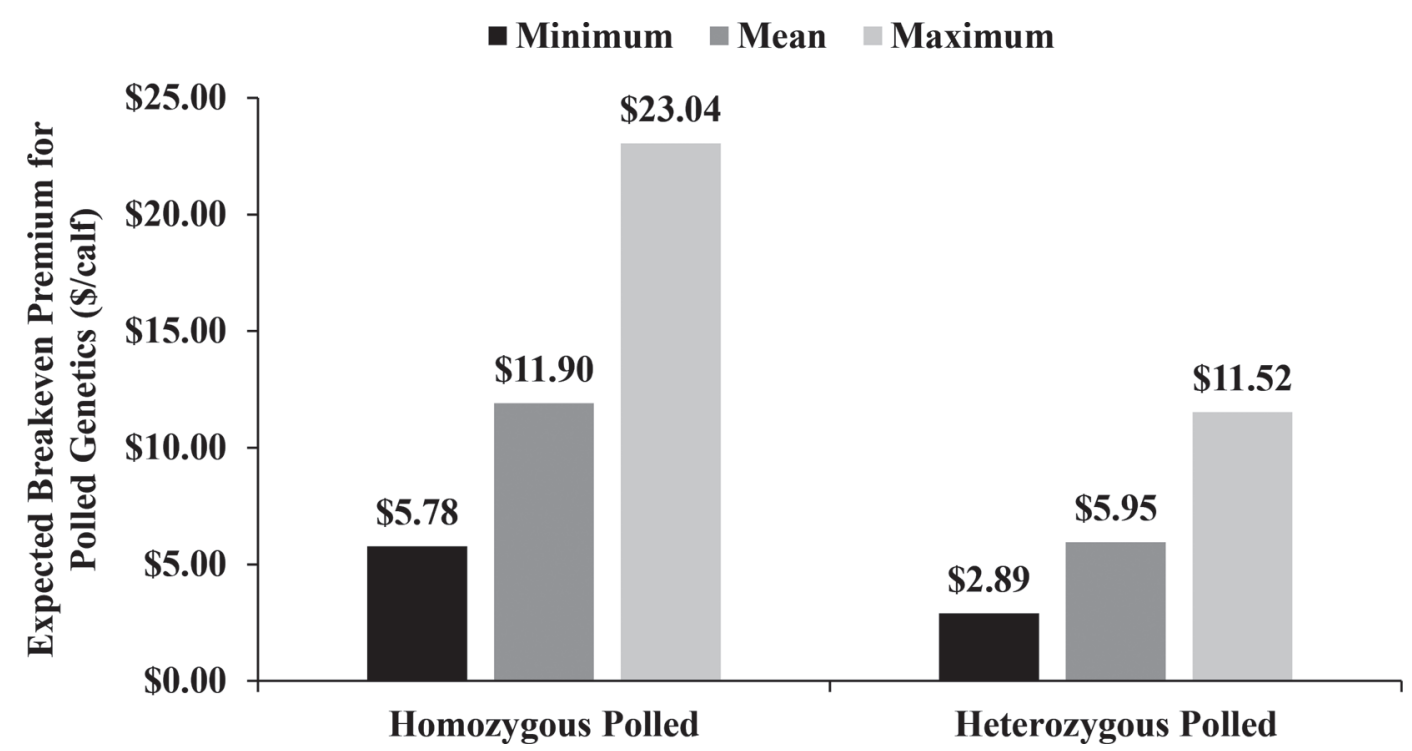

Figure 4. Minimum, mean, and maximum expected breakeven premiums ( $\$ /$ calf) for homozygous and heterozygous polled genetics.

additional $\$ 23 /$ head ( $\$ 8 /$ straw of semen) for homozygous polled genetics and nearly $\$ 12 /$ head ( $\$ 4 /$ straw of semen) for heterozygous polled genetics.

Although the stochastic approach to estimating costs associated with different dehorning methods provides a robust analysis of these alternatives, several assumptions of our model should be considered when interpreting these results. For example, remember that any potential time savings associated with more docile calves during dehorning as a result of the administration of pain relief were not included in our model. Therefore, should this time savings be realized, labor costs for these scenarios would decrease, making the use of pain relief more competitive relative to scenarios that did not include pain relief. In addition, the cost associated with potential unnecessary dehorning of polled animals with scurs was not included in our model. This is important, because excluding this potential cost, although likely small for most producers, means that our model underestimates the true cost of incorporating polled genetics into a breeding program, and therefore may overestimate the breakeven premiums associated with polled genetics.

The assumption that the initial cow herd is $100 \%$ homozygous horned is certainly reasonable (Spurlock et al., 2014) but is also the most conservative approach to estimating the distribution of phenotypes of calves sired by heterozygous polled bulls. Similarly, the model is static, and does not take into account the changing distribution of polled genotypes in the cow herd over time. As more cows carrying the polled gene enter the breeding herd through retention or purchase of polled heifers, the proportion of polled offspring would also increase. In the short run, this would cause the use of heterozygous sires to become more competitive relative to homozygous polled sires from a cost perspective. However, over time, more proactive selection and breeding strategies would be required to manipulate or maintain the distribution of polled genotypes in the herd and meet the overall objectives of the farm (Spurlock et al., 2014; Scheper et al., 2016).

\section{Inferring Economic and Social Benefits of Polled Genetics}

Animal welfare concerns have historically been more commonly associated with Europe, but as the US public has placed increased attention on livestock animal welfare, various industries have been under pressure to reevaluate production processes and management techniques in the United States. Dairy producers, in particular, have faced concern from US residents regarding the welfare implications of 2 common practices - dehorning and tail docking [N. J. O. Widmar, C. Morgan (Purdue University, West Lafayette, IN), C. A. Wolf, E. A. Yeager (Kansas State University, Manhattan), and C. C. Croney (Purdue University); unpublished data]. Although current US regulations neither prohibit nor mandate that pain management be incorporated into dehorning procedures, the potential exists for social, regulatory, or market-based pressures (including mandating of changes in production systems by food retailers or processors) to drive the dairy industry toward change. Schweikhardt and Browne (2001) document and detail "the growth of politics by other means - politics practiced through the market" and provide examples of 
food companies having made decisions that have been influenced by factors that might have been thought to have belonged in the political realm to meet changing consumer demands. In particular, as evidenced by Schweikhardt and Browne (2001) and explored by Olynk et al. (2009), consumers are particularly sensitive about the practices and procedures used to make food products that are consumed by infants and young children. Given the wholesome and pure reputation of dairy foods, and the obvious linkages to the diets of children and infants, it is conceivable that milk and dairy products are particularly sensitive products for US consumers.

Dairy cattle themselves have been previously documented to hold a strong sentiment among US consumers (Olynk et al., 2010). Thus, the public likely places increased attention on the welfare of dairy cows relative to other livestock animals. If calf comfort is prioritized and the market demands that pain relief be incorporated or that dehorning otherwise be regulated, the value of polled genetics is expected to increase beyond the values incorporated into this analysis. Various social aspects of dehorning, including the social acceptance of dehorning using various methods, incorporating pain relief, and the potential for worker aversion to the dehorning process, may become more influential, although the speed and scope of such changes are beyond this analysis. In addition to the speed and scope of such changes, one must consider the duration of incentives. Any potential premiums associated with using polled genetics (as opposed to dehorning) are likely to persist only in the short run. Over time, this premium will likely dissipate as adoption becomes widespread or incorporation of the technology becomes the expectation for market access, rather than a premium factor, such as was the case for recombinant bST, for example.

\section{Industry Challenges and Genetic Advancement}

It is difficult to substantially increase the frequency of polled Holsteins in the population using selection indices that place plausible values on polledness because of its low allele frequency, and even the use of indices that place unreasonably high value on polled is ineffective (Cole, 2015). At present, most farmers do not believe, as evidenced by the semen they select for use in their herds, that the value of polled is worth sacrificing genetic trend in their cows. This perception could change rapidly in response to new consumer demands or regulatory requirements.

An alternative to the use of traditional breeding to increase the frequency of the polled allele in dairy cattle is the use of gene editing to produce high-genetic-merit animals that also are polled. Carlson et al. (2016) re- cently reported on the use of transcription activatorlike effector nucleases (TALENs) to produce 2 healthy, homozygous polled animals. Simulation studies also have shown that the use of gene editing is the most effective way to increase the frequency of polled cattle while maintaining a desirable genetic trend (J. B. Cole, 2015, unpublished data). However, regulatory challenges must be addressed before such animals can enter the food chain. For example, consumers and regulatory agencies have been cautious regarding the entry into the food chain of cloned (USDA, 2008) or genetically engineered (FDA, 2015) animals. The first genetically engineered animal was not approved for entry into the food chain in the United States until 2015 (FDA, 2016). Recently, the USDA Animal and Plant Health Inspection Service determined that a gene-edited white button mushroom is not a regulated article because no foreign DNA was inserted into its genome, although other federal agencies (e.g., FDA) also may have regulatory oversight (USDA, 2016). Although the regulatory situation is unclear with respect to gene-edited animals, there is reason to be optimistic that it eventually will be possible to apply those technologies to food animals. However, as in issues related to animal welfare, public responses to such techniques will vary culturally. For example, genetic modification of plants and animals is prohibited in Europe, whereas methods such as cloning are not seen as problematic in Asia. Ultimately, public opinion of these technologies in the United States has yet to be determined.

If consumer perceptions, market expectations, or social license places further pressure on the dairy industry to eliminate, reduce, or use more precautions in dehorning or disbudding calves, the value of genetic approaches relative to dehorning would increase. As mentioned earlier, due to low allele frequency, even drastic changes in such economic benefits would not alter selection indices (e.g., Net Merit) enough to substantially increase the number of polled individuals. However, recent enhancements to national selection breeding objectives continually shift emphasis from production to other traits of health and management ease (Van Raden and Cole, 2014). Thus, while economic merit of polledness is not likely to allow its value in selection indices to substantially alter allele frequency in the population, inclusion of the value of naturally hornless phenotypes and genotypes in selection indices would allow better genetic decision making, especially for breeders who prefer polled calves.

\section{CONCLUSIONS}

The EC of the 4 traditional dehorning methods evaluated using simplified parameters in this study ranged 
from $\$ 6$ to $\$ 25 /$ head, with a mean EC around $\$ 12$ to $\$ 13 /$ head. The EC of incorporating polled genetics into a breeding program ranged from $\$ 0$ to $\$ 26 /$ head depending on the additional cost associated with polled relative to horned genetics. Estimated breakeven premiums associated with polled genetics indicate that, on average, producers could spend up to $\$ 5.95 /$ head and $\$ 11.90 /$ head more for heterozygous and homozygous polled genetics, respectively, compared with horned genetics (or $\$ 2.08$ and $\$ 4.17 /$ straw of semen at an assumed average conception rate of $35 \%$ ). Given the parameters outlined, sensitivity to individual farms' semen and dehorning costs are likely to swamp these differences. Beyond on-farm costs, industry-wide discussion may be warranted surrounding the public's acceptance and attitude toward polled genetics versus mechanical dehorning or disbudding of calves. The value of avoiding dehorning may be larger for the industry, and perhaps some individual farms, than initially suggested if additional value is put on calf comfort and possible worker aversion to the dehorning process. If public perception of dehorning influences market access, the EC of dehorning may be very large, but that cost is impossible to estimate at present. The results from this analysis point to the potential for economical incorporation of polled genetics into breeding programs, even under current market conditions, depending on the availability, cost, and genetic merit of semen. As the availability and breadth of polled sires continues to increase and the gap in genetic merit between conventional and polled sires continues to close, we would expect more producers to start incorporating polled genetics into their breeding programs. In particular, those producers facing relatively high costs of dehorning or those who are able to capture premiums associated with improved animal welfare may be inclined to pursue incorporation of polled genetics in their herds. However, these improvements can only take place if genetics companies continue to make advancements toward providing highquality polled sires, which would be particularly prudent of companies who anticipate consumer or market acceptance of dehorning to wane.

\section{ACKNOWLEDGMENTS}

Thompson, Widmar, Schutz, and Wolf were supported by internal university discretionary funds. Cole was supported by appropriated project 8042-31000-101-00D, "Improving Genetic Predictions in Dairy Animals Using Phenotypic and Genomic Information," of the Agricultural Research Service of the USDA. Mention of trade names or commercial products in this article is solely for the purpose of providing specific information and does not imply recommendation or endorsement by the USDA.

\section{REFERENCES}

AABP (American Association of Bovine Practitioners). 2014. Castration and dehorning guidelines. Accessed March 3, 2016. http://www.aabp.org/resources/AABP_Guidelines/Castration_ and_Dehorning_Guidelines_app3.2014_03.17.2014.pdf.

AVMA (American Veterinary Medical Association). 2014. Literature review on the welfare implications of the dehorning and disbudding of cattle. Accessed March 3, 2016. https://www.avma.org/KB/ Resources/LiteratureReviews/Pages/Welfare-Implications-ofDehorning-and-Disbudding-Cattle.aspx.

Bates, A. J., P. Eder, and R. A. Laven. 2015. Effect of analgesia and anti-inflammatory treatment on weight gain and milk intake of dairy calves after disbudding. N. Z. Vet. J. 63:153-157.

Bullock, D. 2015. The genetics of horned, polled, and scurred cattle. Accessed Nov. 30, 2016. http://articles.extension.org/sites/ default/files/2015-4_Genetics $\% 20$ of $\% 20$ Horned $\% 20$ Polled $\% 20$ and $\% 20$ Scurred\%20Cattle_Arial.pdf.

Carlson, D. F., C. A. Lancto, B. Zang, E.-S. Kim, M. Walton, D. Oldeschulte, C. Seabury, T. S. Sonstegard, and S. C. Fahrenkrug. 2016. Production of hornless dairy cattle from genome-edited cell lines. Nat. Biotechnol. 34:479-481.

Cole, J. B. 2015. A simple strategy for managing many recessive disorders in a dairy cattle breeding program. Genet. Sel. Evol. 47:94.

Cole, J. B., P. M. VanRaden, J. R. O'Connell, C. P. Van Tassell, T. S. Sonstegard, R. D. Schnabel, J. F. Taylor, and G. R. Wiggans. 2009. Distribution and location of genetic effects for dairy traits. J. Dairy Sci. 92:2931-2946.

Cozzi, G., F. Gottardo, M. Brscic, B. Contiero, N. Irrgang, U. Knierim, O. Pentelescu, J. J. Windig, L. Mirabito, F. Kling Eveillard, A. C. Dockes, I. Veissier, A. Velarde, C. Fuentes, A. Dalmau, and C. Winckler. 2015. Dehorning of cattle in the EU member states: A quantitative survey of the current practices. Livest. Sci. 179:4-11.

Dorshorst, B. 2014. Half of Holstein heifer calves could be polled by 2034. Progressive Dairyman. Accessed Feb. 26, 2017. http://www. progressivedairy.com/topics/a-i-breeding/half-of-holstein-heifercalves-could-be-polled-by-2034.

Ellis, S., and L. Schulz. 2015. Livestock enterprise budgets for Iowa 2015. Iowa State University Extension and Outreach, Ag Decision Maker File B1-21. Iowa State University, Ames.

FDA (Food and Drug Administration). 2015. Genetically engineered animals: General Q\&A. FDA, Animal and Veterinary, Silver Spring, MD. Accessed Sep. 1, 2016. http://www. fda.gov/AnimalVeterinary/Development ApprovalProcess / GeneticEngineering/GeneticallyEngineeredAnimals/ucm113605. htm.

FDA (Food and Drug Administration). 2016. Genetically Engineered Animals: AquAdvantage Salmon. FDA, Animal and Veterinary, Silver Springs, MD. Accessed Sep. 1, 2016. http://www. fda.gov/AnimalVeterinary/Development ApprovalProcess / GeneticEngineering/GeneticallyEngineeredAnimals/ucm280853. htm.

Laden, S. A., J. E. Wohlt, P. K. Zajac, and R. V. Carsia. 1985. Effects of stress from electrical dehorning on feed intake, growth, and blood constituents of Holstein heifer calves. J. Dairy Sci. 68:3026-3066.

Newton, H. P., and A. M. O'Connor. 2013. The economics of pain management. Vet. Clin. North Am. Food Anim. Pract. 29:229-250.

Olynk, N. J., G. T. Tonsor, and C. A. Wolf. 2010. Consumer willingness to pay for livestock credence attribute claim verification. J. Agric. Resour. Econ. 35:261-280.

Olynk, N. J., and C. A. Wolf. 2008. Economic analysis of reproductive management strategies on US commercial dairy farms. J. Dairy Sci. 91:4082-4091.

Olynk, N. J., and C. A. Wolf. 2009. Stochastic economic analysis of dairy cattle artificial insemination reproductive management programs. J. Dairy Sci. 92:1290-1299. 
Olynk, N. J., C. A. Wolf, and G. T. Tonsor. 2009. Labeling of credence attributes in livestock production: Verifying attributes which are more than "meet the eye." J. Food Law Pol. Rev. 5:181-200.

Payne, M. 2016. California dairy quality assurance program (CDQAP) ruminations: Dehorning and economics. California Dairy Research Foundation. Accessed Mar. 3, 2016. http://cdrf. org/2016/01/06/5046/.

Scheper, C., M. Wensch-Dorendorf, T. Yin, H. Dressel, H. Swalve, and S. König. 2016. Evaluation of breeding strategies for polledness in dairy cattle using a newly developed simulation framework for quantitative and Mendelian traits. Genet. Sel. Evol. 48:50.

Schweikhardt, D. B., and W. P. Browne. 2001. Politics by other means: The emergence of a new politics of food in the United States. Rev. Agric. Econ. 23:302-318.

Spurlock, D. M., M. L. Stock, and J. F. Coetzee. 2014. The impact of 3 strategies for incorporating polled genetics into a dairy cattle breeding program on the overall herd genetic merit. J. Dairy Sci. 97:5265-5274.

Stafford, K. J., and D. J. Mellor. 2005. Dehorning and disbudding distress and its alleviation in calves. Vet. J. 169:337-349.

STgenetics. 2016. Fall 2016 Holstein sire summaries. Accessed Nov. 30, 2016. http://www.stgen.com/default.aspx?language=english.

Stock, M. L., S. L. Baldridge, D. Griffin, and J. F. Coetzee. 2013. Bovine dehorning: Assessing pain and providing analgesic management. Vet. Clin. North Am. Food Anim. Pract. 29:103-133.

USDA. 2008. FDA's final risk assessment, management plan and industry guidance on animal clones and their progeny. USDA: Office of Communications, Washington D.C. Release No. 0011.08. Accessed Sep. 1, 2016. http://www.usda.gov/wps/portal/usda/ usda mediafb? contentid $=2008 / 01 / 0011 . x m l \&$ printable $=$ true.

USDA. 2009. Dairy 2007, Part IV: Reference of dairy cattle health and management practices in the United States, 2007. USDA Animals and Plant Health Inspection Service. Fort Collins, CO. \#N494.0209. Accessed Mar. 3, 2016. https://www.aphis.usda.gov/ animal_health/nahms/dairy/downloads/dairy07/Dairy07_dr_ PartIV.pdf.

USDA. 2016. Re: Request for confirmation that transgene-free, CRISPR-edited mushroom is not a regulated article. USDA Animal and Plant Health Inspection Service, Riverdale, MD. Accessed Sep. 1, 2016. https://www.aphis.usda.gov/biotechnology/ downloads/ reg_loi/15-321-01_air_response_signed.pdf.

Van Raden, P. M., and J. B. Cole. 2014. Net Merit as a measure of lifetime profit: 2014 revision. Animal Improvement Program, Animal Genomics and Improvement Laboratory, Agricultural Research Service, USDA, Beltsville, MD. Accessed Aug. 23, 2016. http:// aipl.arsusda.gov/reference/nmcalc-2014.htm.

Vickers, K. J., L. Niel, L. M. Kiehlbauch, and D. M. Weary. 2005. Calf response to caustic paste and hot-iron dehorning using sedation with and without local anesthetic. J. Dairy Sci. 88:1454-1459.

Widmar, N. J. O., M. M. Schutz, and J. B. Cole. 2013. Breeding for polled dairy cows versus dehorning: Preliminary cost assessments and discussion. J. Dairy Sci. 96(E. Suppl. 1):602 (Abstr. TH373).

Windig, J. J., R. A. Hoving-Boling, and R. F. Veerkamp. 2015. Breeding for polledness in Holstein cattle. Livest. Sci. 179:96-101. 